\title{
End-to-end correlated subgap states in hybrid nanowires
}

\author{
G. L. R. Anselmetti, ${ }^{1}$ E. A. Martinez,${ }^{1}$ G. C. Ménard,${ }^{1}$ D. Puglia, ${ }^{1}$ F. K. Malinowski,${ }^{1}$ J.S. Lee,${ }^{2}$ S. Choi, ${ }^{2}$ \\ M. Pendharkar, ${ }^{3}$ C. J. Palmstrøm, ${ }^{2,3,4}$ C. M. Marcus, ${ }^{1}$ L. Casparis, ${ }^{1, \text { * }}$ and A. P. Higginbotham ${ }^{1,5, \dagger}$ \\ ${ }^{1}$ Center for Quantum Devices, Niels Bohr Institute, \\ University of Copenhagen, and Microsoft Quantum - Copenhagen, \\ Universitetsparken 5, 2100 Copenhagen, Denmark \\ ${ }^{2}$ California NanoSystems Institute, University of California, Santa Barbara, California 93106, USA \\ ${ }^{3}$ Department of Electrical Engineering, University of California, Santa Barbara, California 93106, USA \\ ${ }^{4}$ Materials Department, University of California, Santa Barbara, California 93106, USA \\ ${ }^{5}$ Institute of Science and Technology Austria, 3400 Klosterneuburg, Austria
}

(Dated: September 18, 2019)

\begin{abstract}
End-to-end correlated bound states are investigated in superconductor-semiconductor hybrid nanowires at zero magnetic field. Peaks in subgap conductance are independently identified from each wire end, and a cross-correlation function is computed that counts end-to-end coincidences, averaging over thousands of subgap features. Strong correlations in a short, $300 \mathrm{~nm}$ device are reduced by a factor of four in a long, $900 \mathrm{~nm}$ device. In addition, subgap conductance distributions are investigated, and correlations between the left and right distributions are identified based on their mutual information.
\end{abstract}

Single Majorana bound states emerge at each end of a one-dimensional topological superconductor 1], and pairs of Majorana bound states have been proposed to nonlocally encode quantum information [2, 3. Following the theoretical suggestion that hybrid superconductorsemiconductor nanowires can possess a topological phase [4, 5], bound states within the superconducting gap (subgap states), have been extensively studied using the tunneling-conductance from a single wire end, and the results are broadly consistent with Majorana modes [ 6 10. It has also been discovered, both in experiment [11, 12 and theory [13 19, that localized non-topological or quasi-Majorana bound states can mimic many signatures of well-separated Majorana bound states. Further, quantum-dot experiments can give information on the spatial extent of subgap states [12, 20,22. Probing both ends of the Majorana wire has been proposed to distinguish local states from Majorana modes by revealing end-to-end correlations between Majorana pairs, and bulk signatures of the topological transition [19, 23 27.

Independent tunneling spectroscopy of both wire ends - which is fundamentally required to assess the presence of end-to-end correlations - has so far been challenging due to an inability to non-invasively ground the wire bulk. Top-down lithography can interfere with the proximity effect 28, 29, and semiconductor T-junctions have a poorly understood effect on the topological phase. The recently-demonstrated selective area growth (SAG) of superconductor-semiconductor heterostructures offers an appealing solution to this problem, as it allows the lithographic processing on epitaxial contacts to be isolated from the delicate superconductor-semiconductor interface [30, 31]. More broadly, SAG constitutes a potential platform for multi-terminal superconductorsemiconductor devices such as qubits 32 35], Cooperpair splitters [36, and multi-terminal Josephson junc- tions [37 41].

In this Letter, we demonstrate that many of the challenges associated with studying end-to-end correlations in Majorana nanowires have been overcome. We identify subgap features at both ends of three-terminal SAG (a)

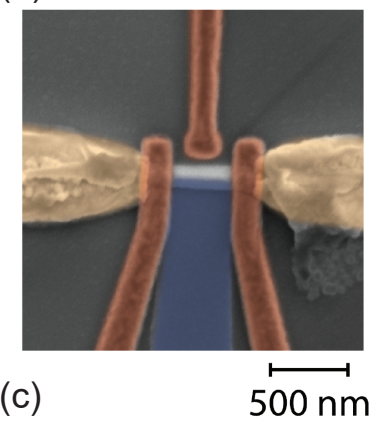

(b)

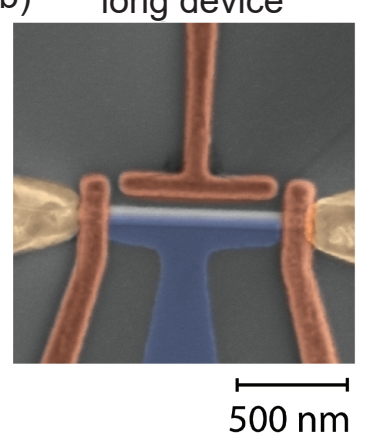

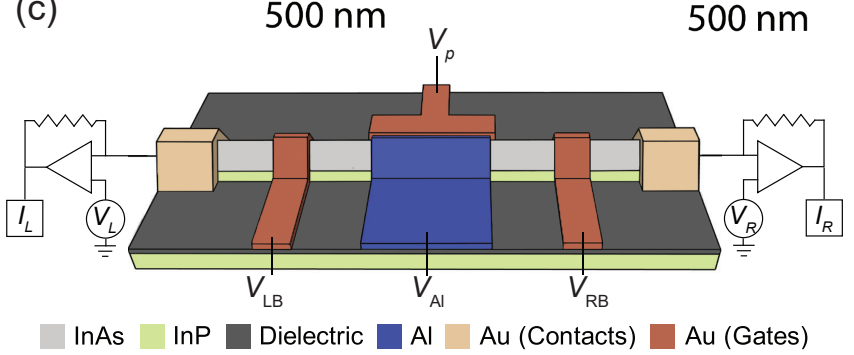

FIG. 1. (a) Scanning electron micrograph of the short device with $300 \mathrm{~nm}$ superconducting segment. (b) Scanning electron micrograph of the long device with $900 \mathrm{~nm}$ superconducting segment. (c) Conceptual sketch of the devices showing the InP substrate, $\mathrm{SiOx}$ mask, $\mathrm{Al}$ lead (blue), Ti/Au Ohmic contacts (yellow), and $\mathrm{Ti} / \mathrm{Au}$ electrostatic gates (red) separated by an HfO layer. Left bias voltage, $V_{L}$, left measured current, $I_{L}$, right bias voltage, $V_{R}$, right measured current, $I_{R}$, aluminum voltage, $V_{\mathrm{Al}}$, left-barrier voltage, $V_{\mathrm{LB}}$, right barrier voltage, $V_{\mathrm{RB}}$, and plunger voltage, $V_{P}$, labeled. 
devices at zero magnetic field, and quantify the correlations by averaging over thousands of subgap features. Varying the length of the superconductor-semiconductor hybrid segment, we find that correlations are reduced by a factor of four in a long, $900 \mathrm{~nm}$ nanowire, as compared to a short, $300 \mathrm{~nm}$ nanowire. We also study the mutual information between the left and right subgap conductance distributions, finding subtle signatures of conductance correlations which are especially pronounced for a family of stable, zero-energy features. This work demonstrates an experimental protocol for quantifying end-toend correlations and determining the associated length scales. Application of these results at non-zero magnetic field will enable searches for correlated Majorana pairs with statistical significance.

The platform for observing end-to-end correlations is Al-InAs SAG, grown by chemical beam epitaxy (CBE) on an InP substrate with a graded InAsP buffer layer [42, with epitaxial Al deposited in situ immediately after semiconductor growth. Following growth, Al (blue Fig. 1) was selectively removed from the substrate and the wire ends by a wet etch, and $\mathrm{Ti} / \mathrm{Au}$ Ohmic contacts [yellow Fig. 1] were deposited on both ends of the wire. The device was then covered by a global HfOx dielectric before electrostatic Ti/Au gates [red Fig. 1] are deposited. Three devices were cofabricated, nominally differing only in the size of the superconducting region. The short [300 nm, Fig. 1(a)] and long [900 nm, Fig. 1(b)] devices were studied in detail. An intermediate-length device did not show evidence of superconductivity and was damaged due to electrostatic discharge; it was not studied further. All measurements were performed in a dilution refrigerator with base electron temperature $<100 \mathrm{mK}$. To focus solely on the identification of endto-end correlations without reference to the topological regime, the magnetic field is fixed to $B=0$; finite-field effects are explored in a partner paper [43.

Both devices have three terminals for electrical measurement [Fig. 1(c)]. Two normal-conducting contacts were used for tunneling spectroscopy, and were equipped with Basel SP983 I-to-V converters $(<100 \Omega$ input impedance). The left normal contact sources a voltage $V_{L}$ and measures a current $I_{L}$. Likewise, the right normal contact sources a voltage $V_{R}$ and measure a current $I_{R}$. The third terminal is formed from a selective etch of the epitaxial $\mathrm{Al}$, and is set to a voltage $V_{\mathrm{Al}}$. Because the third terminal is fabricated from a subtractive process after growth, it can be formed without disrupting the fragile Al-InAs interface. Electrostatic gates, $V_{\mathrm{LB}}$ and $V_{\mathrm{RB}}$, tune the coupling to the left and right leads, and a plunger gate, $V_{P}$, tunes the electrochemical potential within the wire. This setup allows the left conductance, $g_{L}=\delta I_{L} / \delta\left(V_{L}-V_{\mathrm{Al}}\right)$, and the right conductance, $g_{R}=\delta I_{R} / \delta\left(V_{R}-V_{\mathrm{Al}}\right)$ to be independently measured using lock-in techniques. The barriers are adjusted such that the above-gap conductance, averaged over gate volt-
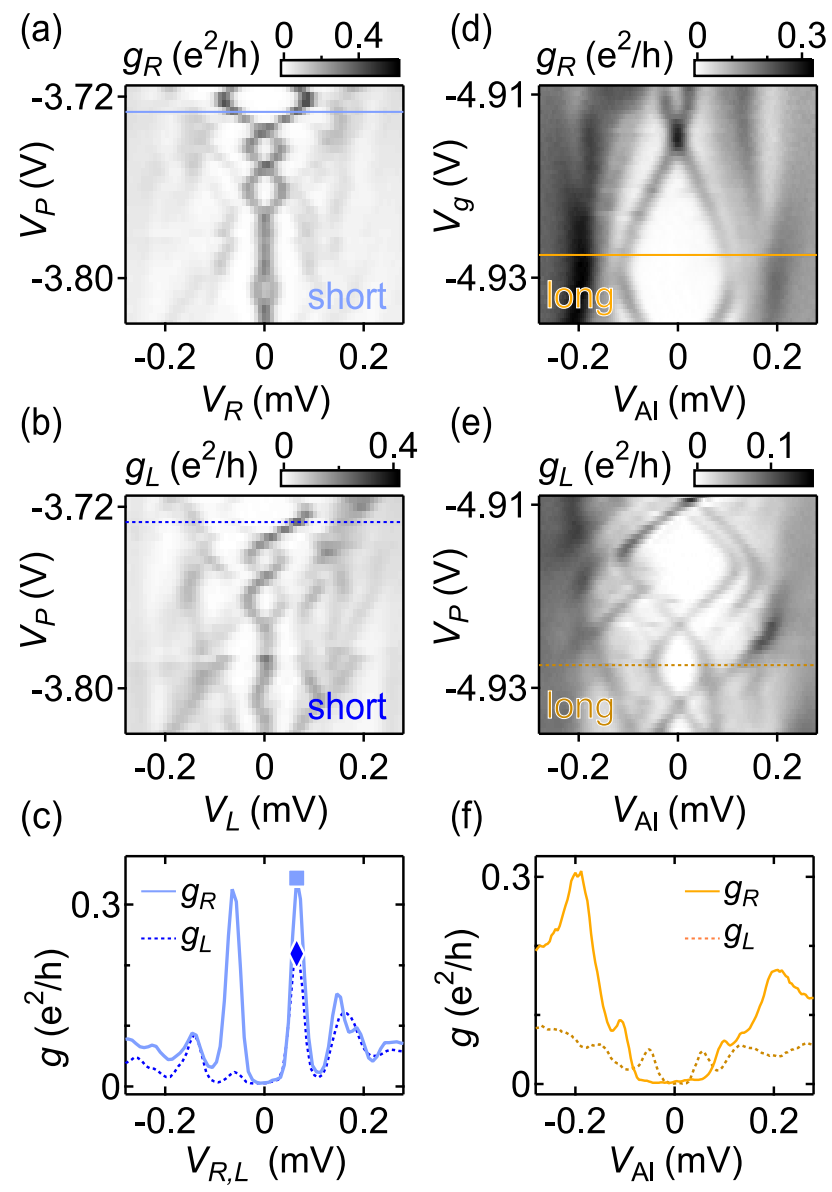

FIG. 2. (a) Measured right conductance, $g_{R}$, versus plunger, $V_{p}$, and right bias, $V_{R}$. (b) Measured left conductance, $g_{L}$, versus plunger, $V_{p}$, and left bias, $V_{L}$. (c) Left and right conductance measured at fixed plunger voltage indicated by horizontal lines in Fig. 1(a,b). $V_{R, L}$ denotes $V_{L}$ for $g_{L}$, and $V_{R}$ for $g_{R}$. Data are from the short device. (d) Measured right conductance, $g_{R}$, versus plunger, $V_{p}$, and middle lead bias, $V_{A l}$. (e) Measured right conductance, $g_{R}$, versus plunger, $V_{p}$, and middle lead bias, $V_{A l}$. (f) Left and right conductance measured at fixed plunger voltage indicated by horizontal lines in Fig. $1(\mathrm{~d}, \mathrm{e}) . V_{R, L}$ denotes $V_{L}$ for $g_{L}$, and $V_{R}$ for $g_{R}$. Data are from the long device.

age, is $<0.15 \mathrm{e}^{2} / \mathrm{h}$.

To study subgap structures in the short device, the right and left conductance, $g_{R}$ and $g_{L}$, were measured as a function of bias and plunger gate with $V_{\mathrm{Al}}=0$ fixed. The data were obtained by sweeping $V_{R}$ while measuring $g_{R}$ with $V_{L}=0$ fixed, and then sweeping $V_{L}$ while measuring $g_{L}$ with $V_{R}=0$ fixed. After these sequential bias sweeps, $V_{P}$ was incremented and the process was repeated. The resulting right-side tunneling conductance [Fig. 2(a)] exhibits a region of suppressed conductance at low bias, a characteristic of a superconductor, with discrete features inside the gap that oscillate as a function of plunger gate, a characteristic of Andreev bound states. The left-side tunneling conductance [Fig. 2(b)] 
(a)

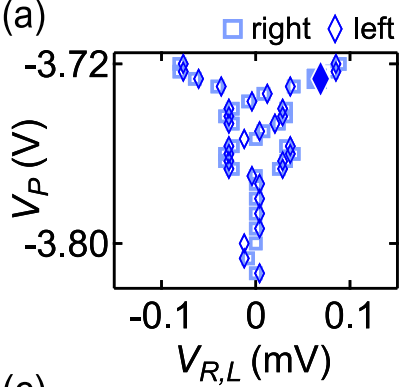

(c)

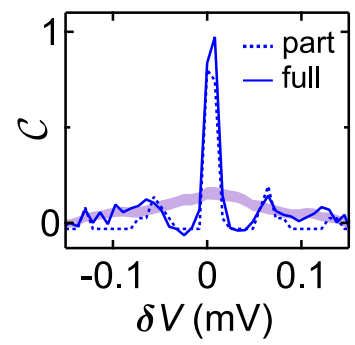

(b)

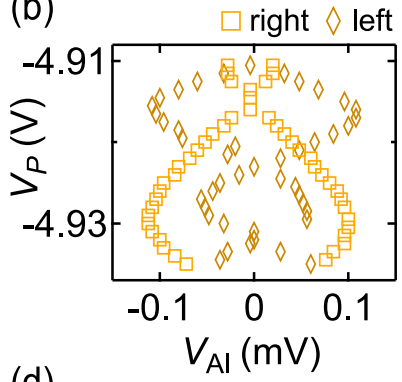

(d)

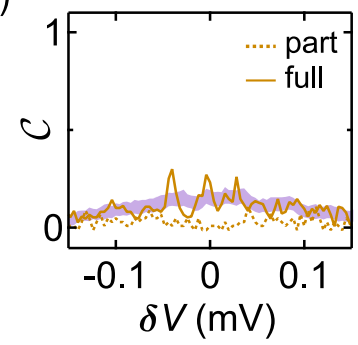

FIG. 3. (a) Extracted conductance-peak plunger voltage, $V_{P}$, and bias voltage, $V_{R, L}$ for the two lowest-energy states from short-device data in Fig. 2(a) [squares] and Fig. 2(b) [diamonds]. Solid square and diamond markers denote points indicated in Fig. 2(c). (b) Extracted conductance-peak plunger voltage, $V_{P}$, and bias voltage, $V_{\mathrm{Al}}$ for the two lowestenergy states from long-device data in Fig. 2(d) [squares] and Fig. 2(e) [diamonds]. Solid square and diamond markers denote points indicated in Fig. 2(f). (c) Correlator $\mathcal{C}$ of the binary peak masks for the data in Fig. 3a [dashed] and the full 2353-peak dataset [solid] from the short device. (d) Correlator $\mathcal{C}$ of the binary peak masks for the data in Fig. $3 \mathrm{~b}$ [dashed] and the full 2058-peak dataset [solid] from the long device. Shaded regions represent $\pm 1 \sigma$ error bands derived from plunger-shifted data.

likewise exhibits a region of suppressed conductance at low bias, with discrete oscillating features inside the gap. The subgap structure observed on the left and right sides are similar. Directly comparing $g_{L}$ and $g_{R}$ at fixed gate voltage, as in Fig. 2(c), emphasizes that subgap peaks generally occur at the same bias voltage, suggesting that left and right peaks originate from the same Andreev bound state. Although peaks are generally observed at the same bias voltages, the conductance associated with these peaks does not show a qualitatively clear correspondence. On both the left and right side, the subgap conductance is not a symmetric function of bias, which is the focal point of a partner paper [43].

It is interesting to note that the low-bias features in Figs. 2(a,b) are isolated from higher-lying states, stable at zero energy, and present on both ends of the device signatures traditionally associated with Majorana modes. We emphasize that the data are taken at $B=0$, where topological effects are not generally expected [4, 5].

Turning to the long device, $g_{R}$ versus gate and bias exhibits a gapped low-bias conductance with discrete subgap features [Fig. 2(d)], qualitatively similar to the short

(a)

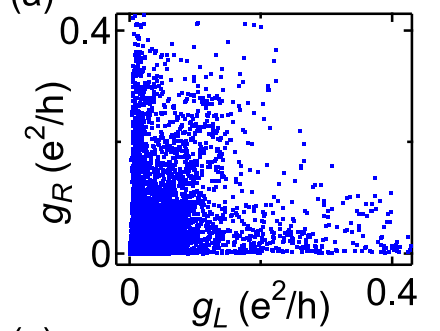

(b)
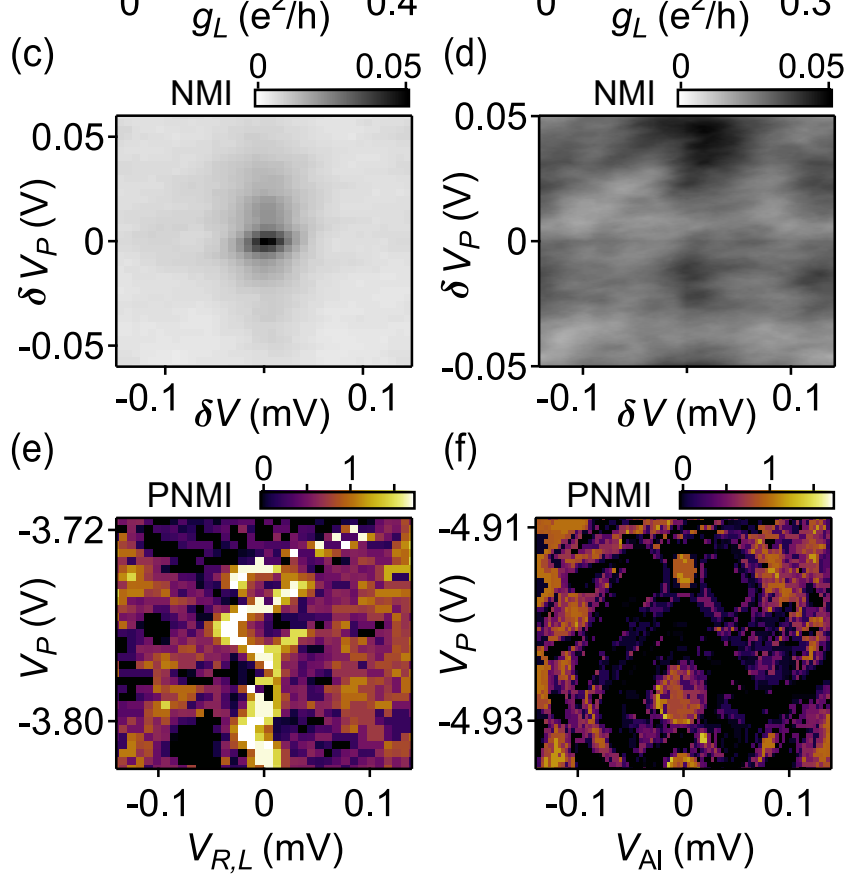

(f)
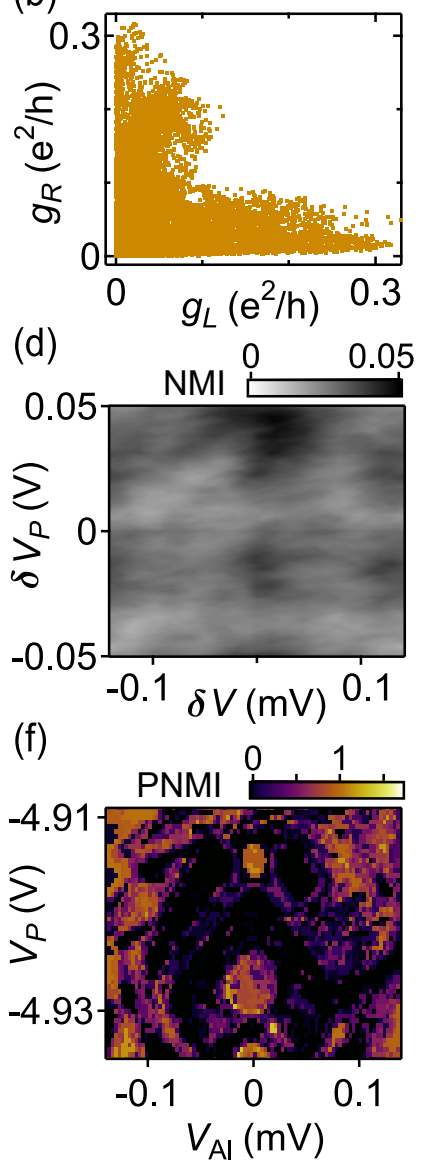

FIG. 4. Sub-gap conductance pairs $\left(g_{R}, g_{L}\right)$ for short device bias $V_{R, L}<0.15 \mathrm{mV}$ (a) and long device bias $V_{\mathrm{Al}}<0.15 \mathrm{mV}$ (b). Normalized mutual information, NMI, of the joint conductance distribution as a function bias-shift, $\delta V$, and plunger shift, $\delta V_{P}$ for the short device (c) and long device (d). Pointwise normalized mutual information, PNMI, for short device subgap states (a) and for long device subgap states (b).

device. The left-side conductance, shown in Fig. 2(e), also exhibits a gapped low-bias conductance and discrete subgap features. Unlike the short device, in the long device the left and right subgap features exhibit markedly different gate-voltage dependences. A direct comparison of $g_{L}$ and $g_{R}$ for the long device, shown in Fig. 2(f), confirms that subgap peaks on the left and right occur at different bias voltages, suggesting that the subgap features originate from different Andreev bound states. We note that the long-device data are acquired differently than the short device data; by sweeping the aluminum bias $V_{\mathrm{Al}}$ and simultaneously measuring the currents on the left and right with $V_{R, L}=0$. This method has the advantage of simultaneous data acquisition on the left and right sides, but is disadvantageous in the short device, where the left and right biases must be carefully trimmed to zero to avoid complications from nonlocal effects. We have directly compared the two methods on a 
small subset of the data and found that they are in agreement [44. Taken together, the observations in Fig. 2 suggest a picture where individual subgap states can extend across the short device, but are associated with a single end of the long device.

To further analyze the conductance data, subgap peaks are identified using a peak finding algorithm over a wide range of gate voltages, of which the data in Fig. 2 is a small subset. A total of 2353 peaks are extracted from the full short device dataset, and 2058 peaks are extracted from the full long device dataset. A plot of the identified peak locations for each plunger-gate value from Fig. 2 demonstrates that the peaks are identified accurately, and emphasizes that the sugap peaks are indeed generally correlated for the short device [Fig. 3(a)], but are uncorrelated for the long device [Fig. 3(b)].

The correlations are quantified by introducing binary peak masks for the left side, $B_{L}\left(V, V_{P}\right)$, and the right side, $B_{R}\left(V, V_{P}\right)$ which take a value of 1 if a peak is identified at bias voltage $V$ and plunger voltage $V_{P}$ and a value of 0 otherwise. The cross-covariance, $\mathcal{C}$, between the left and right binary masks is then computed, allowing for a bias offset, $\delta V$, between the two sides,

$$
\begin{aligned}
\mathcal{C}(\delta V)= & \left\langle B_{L}\left(V, V_{P}\right) B_{R}\left(V+\delta V, V_{P}\right)\right\rangle \\
& -\left\langle B_{L}\left(V, V_{P}\right)\right\rangle\left\langle B_{R}\left(V, V_{P}\right)\right\rangle,
\end{aligned}
$$

where $\langle\cdot\rangle$ denotes the expectation value with respect to both $V$ and $V_{P}$. $\mathcal{C}$ serves as a correlation metric by counting the number of coincident peaks in each bias trace, averaged over plunger voltage.

In the short device, $\mathcal{C}$ is peaked around $\delta V=0$. Including only the subset of data from Fig. 3(a) results in a correlator that is significantly less than unity [dashed trace, Fig. 3(c)], indicating that on average less than one peak is identified as being correlated at each gate voltage. This is a result of the stringent definition of $\mathcal{C}$; it counts peaks as correlated only if they occur at identical bias voltages. Indeed, inspection of the data in Fig. 3(a) reveals that every peak has a nearby partner, while only some occur at identical parameters. Computing $\mathcal{C}$ for the full short-device dataset [solid trace, Fig. 3(c)] increases the average number of coincident subgap peaks to $\mathcal{C}=0.97$. For large bias shifts, the correlator $\mathcal{C}$ has small fluctuations, consistent with the background level inferred by introducing a gate-voltage shift between the datasets.

In contrast, for the long device, no significant correlations are observed from the subset of dataset in Fig. 3(b) [dashed trace, Fig. 3(d)], and a small correlation peak of $\mathcal{C}=0.27$ is resolved when averaging over the full dataset [solid trace, Fig. 3(d)]. The observed drop in correlations, by a factor of 3.7, presumably reflects a characteristic length-scale for subgap states in these structures. By examining subsets of the long device dataset, we have identified a small region in gate voltage that gives rise to the correlations 44, demonstrating that $\mathcal{C}$ is a useful tool for identifying rare features in the data.

While the presence of subgap features in the short device is strongly correlated between both ends, the conductance associated with these features fluctuates strongly. To study correlations in these fluctuations, each measurement of the right and left conductances is visualized as a point in the $\left(g_{R}, g_{L}\right)$ plane. The subgap $\left(g_{R}, g_{L}\right)$ data for the short device [Fig. 4(a)] is indeed widely distributed, with a long tail extending out to $g_{R, L} \sim 0.4 \mathrm{e}^{2} / \mathrm{h}$. In the long device, the subgap $\left(g_{R}, g_{L}\right)$ data are also broadly scattered, but the $\left(g_{R}, g_{L}\right)$ pairs tend more towards the axes in the $g_{R}, g_{L}$ plane [Fig. 4(b)]. In other words, there are more points with large weight on both sides in the short device as compared to the long device, which gives a preliminary indication of positive correlations in conductance for the short device.

To quantify this observation, joint distributions, $P\left(g_{R}, g_{L}\right)$, and marginal distributions, $P\left(g_{R}\right)$ and $P\left(g_{L}\right)$, are estimated from the scatter-plot data. In general, for independent variables one expects $P(a, b)=P(a) P(b)$. Deviations from this relationship are quantified by the normalized mutual information, NMI, which, in analogy with a correlator, is calculated as a function of bias and plunger shift between the left and right datasets, $\mathrm{NMI}=\mathrm{NMI}\left(\delta V, \delta V_{P}\right)[44$.

For the short device, the mutual information is strongly peaked at $\delta V=\delta V_{P}=0$ [Fig. 4(c)], indicating the presence of correlations in the conductance distributions. As a function of bias shift, $\delta V$, the mutual information decays with a $1 / e$ characteristic length that approximately matches the observed width of subgap peaks, suggesting that the conductance correlations are associated with subgap states. As a function of plunger shift, $\delta V_{P}$, the mutual information also decays sharply, again consistent with contributions from subgap states. In contrast, for the long device, the mutual information is relatively flat and featureless [Fig. 4(d)], suggesting that there are no conductance correlations originating from the subgap states. The mutual information for the long device, however, still differs significantly from zero, indicating there are broad, gate and bias-voltage independent features that are mutually dependent between the two ends.

A more granular view of the conductance correlations is obtained by visualizing the pointwise normalized mutual information, PNMI, which is defined by the relation $\mathrm{NMI}=\overline{\mathrm{PNMI}}$, where the overline denotes an expectation value over $P\left(g_{R}, g_{L}\right)$. Whereas NMI is a property of the entire distribution, PNMI is associated with individual $\left(g_{R}, g_{L}\right)$ pairs, and can therefore be mapped as a function of the measurement parameters. In the short device, a map of the PNMI as a function of gate and bias voltage reveals regions of elevated PNMI corresponding to the stable low-lying bound state identified in previous dis- 
cussion [Fig. 4(e)]. This bound state also has an elevated PNMI compared to other correlated features throughout the entire dataset 44, suggesting that although many features in the short device are measurable from both device ends, additional correlations in the conductance of these features are relatively rare. In contrast to the short device, for the long device the PNMI is smaller, although there is still some weak structure [Fig. 4(f)]. The conductance correlations are not thoroughly understood, although, qualitatively, correlations in the conductance could be expected as a result of states that span the entire device.

In summary, we interpret the well-correlated subgap states in the short device as a clear indication that bound states extend several hundred nanometers into the hybrid region, essentially spanning the entire device. If the zero-field bound states are attributed to an accidental potential well in the tunneling region [9], then their spatial extent would indicate a soft confinement, which will strongly effect the behaviour at nonzero magnetic field [13, 14, 17. Following this reasoning, we anticipate that these measurements could be used to refine the theoretical understanding of topologically trivial subgap states, including in numerical simulations, and possibly aid in distinguishing them from the topological case.

Looking ahead, the techniques developed here can be applied in the putative topological regime. Computing a correlator after feature extraction, as in Fig. (3), effectively removes the background from conductance fluctuations. Quantifying conductance-correlations using the mutual information requires minimal analysis of the data, and explicitly tests for correlations in the conductance values of all data points.

We thank Karsten Flensberg, Martin Leijnse, Dmitry Pikulin, Roman Lutchyn, and Gašper Tkačik for valuable discussions. Research was supported by Microsoft Corporation and the Danish National Research Foundation.

* Equal contribution, lucas.casparis@microsoft.com

$\dagger$ Equal contribution, andrew.higginbotham@ist.ac.at

[1] A. Y. Kitaev, Physics-Uspekhi 44, 131 (2001)

[2] A. Kitaev, Annals of Physics 303, 2 (2003)

[3] C. Nayak, S. H. Simon, A. Stern, M. Freedman, and S. Das Sarma, Rev. Mod. Phys. 80, 1083 (2008).

[4] R. M. Lutchyn, J. D. Sau, and S. Das Sarma, Phys. Rev. Lett. 105, 077001 (2010).

[5] Y. Oreg, G. Refael, and F. von Oppen, Phys. Rev. Lett. 105, $177002(2010)$

[6] V. Mourik, K. Zuo, S. M. Frolov, S. R. Plissard, E. P. A. M. Bakkers, and L. P. Kouwenhoven, Science 336, $1003(2012)$

[7] A. Das, Y. Ronen, Y. Most, Y. Oreg, M. Heiblum, and H. Shtrikman, Nat. Phys 8, 887 (2012).

[8] H. O. H. Churchill, V. Fatemi, K. Grove-Rasmussen, M. T. Deng, P. Caroff, H. Q. Xu, and C. M. Marcus,
Phys. Rev. B 87, 241401(R) (2013).

[9] M. T. Deng, S. Vaitiekenas, E. B. Hansen, J. Danon, M. Leijnse, K. Flensberg, J. Nygård, P. Krogstrup, and C. M. Marcus, Science 354, 1557 (2016).

[10] H. Zhang, C.-X. Liu, S. Gazibegovic, D. Xu, J. A. Logan, G. Wang, N. van Loo, J. D. S. Bommer, M. W. A. de Moor, D. Car, R. L. M. Op het Veld, P. J. van Veldhoven, S. Koelling, M. A. Verheijen, M. Pendharkar, D. J. Pennachio, B. Shojaei, J. S. Lee, C. J. Palmstrøm, E. P. A. M. Bakkers, S. Das Sarma, and L. P. Kouwenhoven, Nature 556, 7479 (2018).

[11] E. J. H. Lee, X. Jiang, M. Houzet, R. Aguado, C. M. Lieber, and S. De Franceschi, Nature Nanotechnology 9, $79(2013)$.

[12] M.-T. Deng, S. Vaitiekènas, E. Prada, P. San-Jose, J. Nygård, P. Krogstrup, R. Aguado, and C. M. Marcus, Phys. Rev. B 98, 085125 (2018).

[13] G. Kells, D. Meidan, and P. W. Brouwer, Phys. Rev. B 86, 100503(R) (2012)

[14] E. Prada, P. San-Jose, and R. Aguado, Phys. Rev. B 86, 180503 (2012)

[15] J. Cayao, E. Prada, P. San-Jose, and R. Aguado, Phys. Rev. B 91, 024514 (2015).

[16] P. San-Jose, J. Cayao, E. Prada, and R. Aguado, Scientific Reports 6, 21427 EP (2016)

[17] C.-X. Liu, J. D. Sau, T. D. Stanescu, and S. Das Sarma, Phys. Rev. B 96, 075161 (2017).

[18] A. Vuik, B. Nijholt, A. R. Akhmerov, and M. Wimmer, arXiv e-prints (2018), arXiv:1806.02801 [cond-mat.meshall].

[19] C. Reeg, O. Dmytruk, D. Chevallier, D. Loss, and J. Klinovaja, Physical Review B 98, 245407 (2018).

[20] S. M. Albrecht, A. Higginbotham, M. Madsen, F. Kuemmeth, T. S. Jespersen, J. Nygård, P. Krogstrup, and C. M. Marcus, Nature 531, 206 (2016).

[21] F. Peñaranda, R. Aguado, P. San-Jose, and E. Prada, Phys. Rev. B 98, 235406 (2018).

[22] A. M. Whiticar, A. Fornieri, E. C. T. O'Farrell, A. C. C. Drachmann, T. Wang, C. Thomas, S. Gronin, R. Kallaher, G. C. Gardner, M. J. Manfra, C. M. Marcus, and F. Nichele, arXiv:1902.07085 (2019).

[23] S. Das Sarma, J. D. Sau, and T. D. Stanescu, Phys. Rev. B 86, 220506(R) (2012).

[24] B. M. Fregoso, A. M. Lobos, and S. Das Sarma, Phys. Rev. B 88, 180507(R) (2013).

[25] T. D. Stanescu and S. Tewari, Phys. Rev. B 89, 220507(R) (2014)

[26] T. O. Rosdahl, A. Vuik, M. Kjaergaard, and A. R. Akhmerov, Phys. Rev. B 97, 045421 (2018).

[27] Y.-H. Lai, J. D. Sau, and S. Das Sarma, arxiv:1901.02655 (2019).

[28] P. Krogstrup, N. L. B. Ziino, W. Chang, S. M. Albrecht, M. H. Madsen, E. Johnson, J. Nygård, C. M. Marcus, and T. S. Jespersen, Nature Materials 14, 400 (2015), article.

[29] W. Chang, S. M. Albrecht, T. S. Jespersen, F. Kuemmeth, P. Krogstrup, J. Nygård, and C. M. Marcus, Nature Nanotechnology 10, 232 (2015).

[30] F. Krizek, J. E. Sestoft, P. Aseev, S. Marti-Sanchez, S. Vaitiekènas, L. Casparis, S. A. Khan, Y. Liu, T. Stankevič, A. M. Whiticar, A. Fursina, F. Boekhout, R. Koops, E. Uccelli, L. P. Kouwenhoven, C. M. Marcus, J. Arbiol, and P. Krogstrup, Physical Review Materials 2, 093401 (2018). 
[31] S. Vaitiekenas, A. M. Whiticar, M.-T. Deng, F. Krizek, J. E. Sestoft, C. J. Palmstrøm, S. Marti-Sanchez, J. Arbiol, P. Krogstrup, L. Casparis, and C. M. Marcus, Phys. Rev. Lett. 121, 147701 (2018)

[32] T. W. Larsen, K. D. Petersson, F. Kuemmeth, T. S. Jespersen, P. Krogstrup, J. Nygård, and C. M. Marcus, Phys. Rev. Lett. 115, 127001 (2015).

[33] L. Casparis, M. R. Connolly, M. Kjaergaard, N. J. Pearson, A. Kringhøj, T. W. Larsen, F. Kuemmeth, T. Wang, C. Thomas, S. Gronin, G. C. Gardner, M. J. Manfra, C. M. Marcus, and K. D. Petersson, Nature Nanotechnology 13, 915 (2018)

[34] S. Plugge, A. Rasmussen, R. Egger, and K. Flensberg, New Journal of Physics 19, 012001 (2017).

[35] T. Karzig, C. Knapp, R. M. Lutchyn, P. Bonderson, M. B. Hastings, C. Nayak, J. Alicea, K. Flensberg, S. Plugge, Y. Oreg, C. M. Marcus, and M. H. Freedman, Phys. Rev. B 95, 235305 (2017)

[36] L. Hofstetter, S. Csonka, J. Nygård, and C. Schönenberger, Nature 461, 960 (2009).

[37] B. van Heck, S. Mi, and A. R. Akhmerov, Phys. Rev. B 90, $155450(2014)$.

[38] E. Strambini, S. D'Ambrosio, F. Vischi, F. S. Bergeret, Y. V. Nazarov, and F. Giazotto, Nature Nanotechnology 11, 1055 (2016)

[39] J. S. Meyer and M. Houzet, Phys. Rev. Lett. 119, 136807 (2017)

[40] H.-Y. Xie, M. G. Vavilov, and A. Levchenko, Phys. Rev. B 96, 161406(R) (2017)

[41] N. Pankratova, H. Lee, R. Kuzmin, M. Vavilov, K. Wickramasinghe, W. Mayer, J. Yuan, J. Shabani, and V. E. M. Manucharyan, arXiv:1812.06017 (2018).

[42] J. S. Lee, S. Choi, M. Pendharkar, D. J. Pennachio, B. Markman, M. Seas, S. Koelling, M. A. Verheijen, L. Casparis, K. D. Petersson, I. Petkovic, V. Schaller, M. J. Rodwell, C. M. Marcus, P. Krogstrup, L. P. Kouwenhoven, E. P. Bakkers, and C. J. Palmstrøm, arXiv:1808.04563 (2018)

[43] G. C. Ménard, G. L. R. Anselmetti, E. A. Martinez, D. Puglia, F. K. Malinowski, J. S. Lee, S. Choi, M. Pendhakar, C. J. Palmstrøm, K. Flensberg, C. M. Marcus, L. Casparis, and A. P. Higginbotham, arXiv:1905.05505 (2019).

[44] See Supplement, incl Ref.'s 4548.

[45] G. A. Darbellay and I. Vajda, IEEE Transactions on Information Theory 45, 1315 (1999)

[46] N. X. Vinh, J. Epps, and J. Bailey, Journal of Machine Learning Research 11, 2837 (2010)

[47] A. Kraskov, H. Stögbauer, and P. Grassberger, Phys. Rev. E 69, 16 (2004), arXiv:0305641 [cond-mat]

[48] F. Pedregosa, G. Varoquaux, A. Gramfort, V. Michel, B. Thirion, O. Grisel, M. Blondel, P. Prettenhofer, R. Weiss, V. Dubourg, J. Vanderplas, A. Passos, D. Cournapeau, M. Brucher, M. Perrot, and E. Duchesnay, Journal of Machine Learning Research 12, 2825 (2011). 


\title{
Supplement to "End-to-end correlated subgap states in hybrid nanowires"
}

\author{
G. L. R. Anselmetti, ${ }^{1}$ E. A. Martinez,${ }^{1}$ G. C. Ménard,${ }^{1}$ D. Puglia,${ }^{1}$ F. K. Malinowski, ${ }^{1}$ J.S. Lee,${ }^{2}$ S. Choi,${ }^{2}$ \\ M. Pendharkar, ${ }^{3}$ C. J. Palmstr $\varnothing m,{ }^{2,3}, 4$ C. M. Marcus,${ }^{1}$ L. Casparis,${ }^{1,}{ }^{*}$ and A. P. Higginbotham ${ }^{1, \dagger}$ \\ ${ }^{1}$ Center for Quantum Devices, Niels Bohr Institute, \\ University of Copenhagen, and Microsoft Quantum - Copenhagen, \\ Universitetsparken 5, 2100 Copenhagen, Denmark \\ ${ }^{2}$ California NanoSystems Institute, University of California, Santa Barbara, California 93106, USA \\ ${ }^{3}$ Department of Electrical Engineering, University of California, Santa Barbara, California 93106, USA \\ ${ }^{4}$ Materials Department, University of California, Santa Barbara, California 93106, USA
}

\section{COMPARISON OF DIFFERENT BIASING CONFIGURATIONS}

In the main text, the short-device and long-device datasets use different biasing configurations. Here we show that the two biasing methods give the same results by directly comparing them on the short device. Sweeping the right-bias voltage, $V_{R}$ while measuring $I_{\mathrm{R}}$ with $V_{\mathrm{Al}}=0$ generates a map of subgap states [Fig. S1(a)], which is familiar from the main text. Sweeping the aluminum bias voltage, $V_{\mathrm{Al}}$ while measuring $I_{\mathrm{R}}$ with $V_{\mathrm{R}}=0$ fixed reveals the same conductance features [Fig. S1(b)], but with the dependence on bias voltage inverted, as one would expect from the other side of the right junction being biased. Note that the $V_{R}$ and $V_{\mathrm{Al}}$ scans are interlaced so that switches and device instabilities effect the two plots symmetrically. The strong similarity between Fig. S1(a) and Fig. S1(b) explicitly demonstrates the equivalence of the two biasing configurations.

(a)

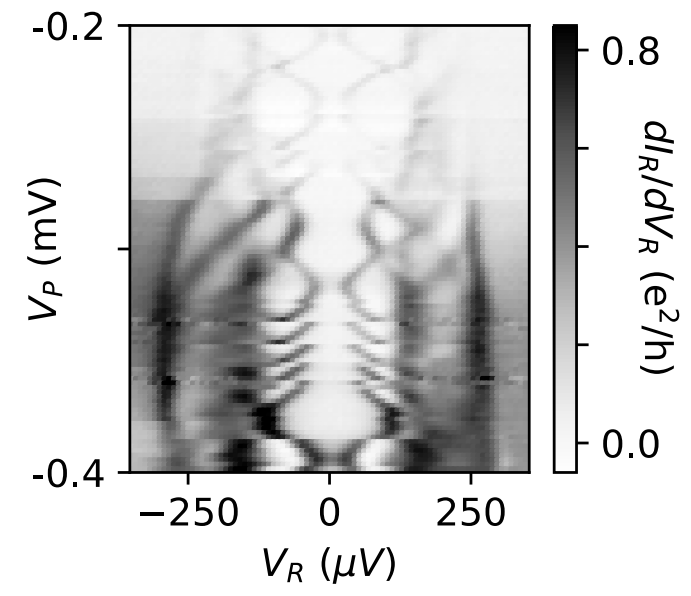

(b)

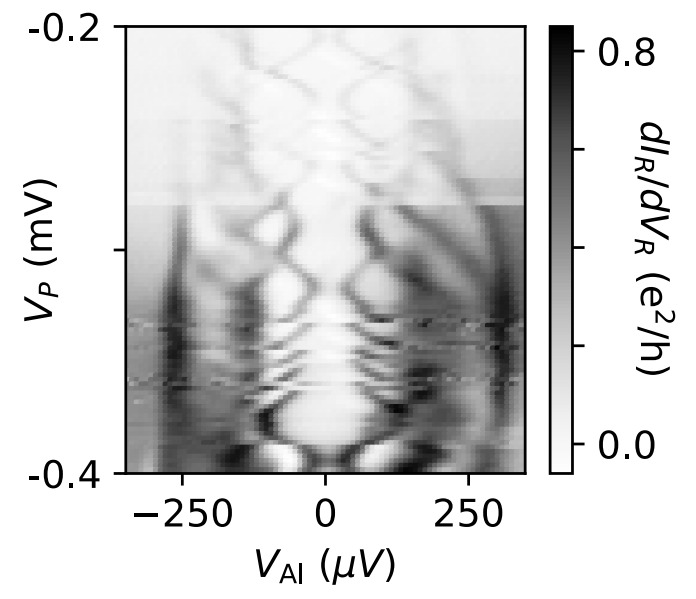

FIG. S1. (a) Right conductance, $d I_{R} / d V_{R}$, measured as a function of right bias, $V_{R}$ and plunger, $V_{P}$. In the main text, this biasing procedure was used for the short device. (b) Right conductance, $d I_{R} / d V_{R}$, measured as a function of aluminum bias, $V_{\mathrm{Al}}$, and plunger, $V_{P}$. In the main text, this biasing procedure was used for the long device.

\section{CORRELATOR ERROR ESTIMATES AND STATISTICAL INFORMATION}

It is interesting to consider the error in the correlation metric, $\mathcal{C}$, that is computed in the main text Fig. 2. One method to estimate the uncertainty is to apply a numerical plunger-voltage shift, $\delta V_{P}$, to one dataset, compute the correlation metric $\mathcal{C}_{\delta V_{P}}$, and then find the standard deviation of $\sigma\left(\mathcal{C}_{\delta V_{P}}\right)$ over a large ensemble of $\delta V_{P}$. Error bands in Fig. 3(c,d) are then given by $\overline{\mathcal{C}_{\delta V_{P}}} \pm \sigma\left(\mathcal{C}_{\delta V_{P}}\right)$.

The computed error, $\sigma\left(\mathcal{C}_{\delta V_{P}}\right)$, is compared with the data for the long-device correlator in Fig. S2. For large bias shifts, the correlator fluctuates but typically remains within the $1 \sigma$ error bars, confirming that the error has been reasonably estimated. At zero bias shift, a $3 \sigma$ peak in $\mathcal{C}$ is observed, with $4 \sigma$ and $3 \sigma$ satellite peaks, confirming that the corrrelations observed in the long device are statistically significant. 


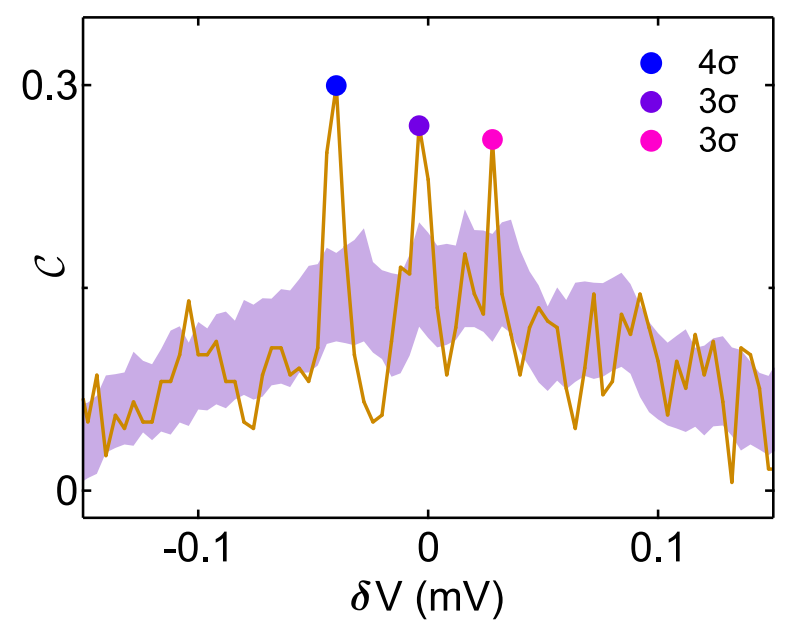

FIG. S2. Correlator, $\mathcal{C}$, for the long-device data set. Shaded region indicates $\pm 1 \sigma$ error bars, estimated from plunger-shifted data. Colored circuls mark a $3 \sigma$ central peak, with $4 \sigma$ and $3 \sigma$ satellite peaks.

When comparing the correlator between the short and long device it is useful to know that the properties of the two datasets are generally similar. The short-device data set has an average of 3.9 subgap peaks per bias scan, and the long-device data set has an average of 5.1 subgap peaks per bias scan. To evaluate the typical autocorrelation of the datasets, we have computed the self-mutual information, $S N M I$, of the conductances as a function of plunger shift, for instance $S N M I\left(\delta V_{P}\right)=N M I\left(g_{R}\left(V_{P}\right), g_{R}\left(V_{P}+\delta V_{P}\right)\right)$. The self-mutual information decays as a function of plunger shift with a characteristic auto-correlation length. The extracted auto-correlation length is $4 \mathrm{mV}$ for the short device, and $1.7 \mathrm{mV}$ for the long device. The resolution on the datasets is one bias scan per $3.3 \mathrm{mV}$ for the short device, and one bias scan per $0.5 \mathrm{mV}$ for the long device.

\section{FULL DATASETS}

For completeness, the full conductance datasets used in the main text are presented in Fig. S3. Most states in the short device can be identified on both sides, albeit with fluctuating weights [Fig. S3(a,b)]. The conductance associated low-lying stable bound state discussed in the main text has an anomalously large point-wise mutual information, $P M I$ [Fig. S3 (c)]. For the long device, most states are uncorrelated, but there is a small region of correlated features responsible for the correlator peak discussed in the main text [orange box Fig. S3(d,e)]. The conductance-PMI for the long device is lower than the short device, with weak structure visible corresponding to the uncorrelated features [Fig. S3(f)].

\section{QUANTIFYING CONDUCTANCE CORRELATIONS WITH MUTUAL INFORMATION}

In Fig. 4(a,b) of the main text we show the simultaneously measured left and right conductances as a scatter plot of $\left(g_{L}, g_{R}\right)$. Our goal is to quantify conductance correlations in this data. The conductances are correlated if they cannot be described by the product of two independent conductance distributions on the left and right sides. The statistical measure for how much the joint conductance distribution deviates from independence is given by the mutual information between the left and right conductances.

In the first place we seek to estimate the joint conductance probability distribution. For this we bin the measured conductance points to calculate a histogram and estimate a discrete probability distribution $p\left(g_{L}, g_{R}\right)$. A uniformly spaced grid is not suitable for our measured conductance data, since the density of points changes by orders of magnitude in different regions of the conductance space. Therefore we use the adaptive binning algorithm of [41], where the sample space is recursively divided in rectangular bins with an equal number of points. As a criterion for continuing the subdivision of a bin, we require there be a minimum number of points in the bin. For the analysis in Fig. 4 we stop subdividing bins with fewer than 80 points, which yields an average of 36 points per bin for the final subdivision. The end result of the algorithm is a grid of $M$ rectangles $A_{i} \times B_{i}$. The probability density $p\left(A_{i} \times B_{i}\right)$ 

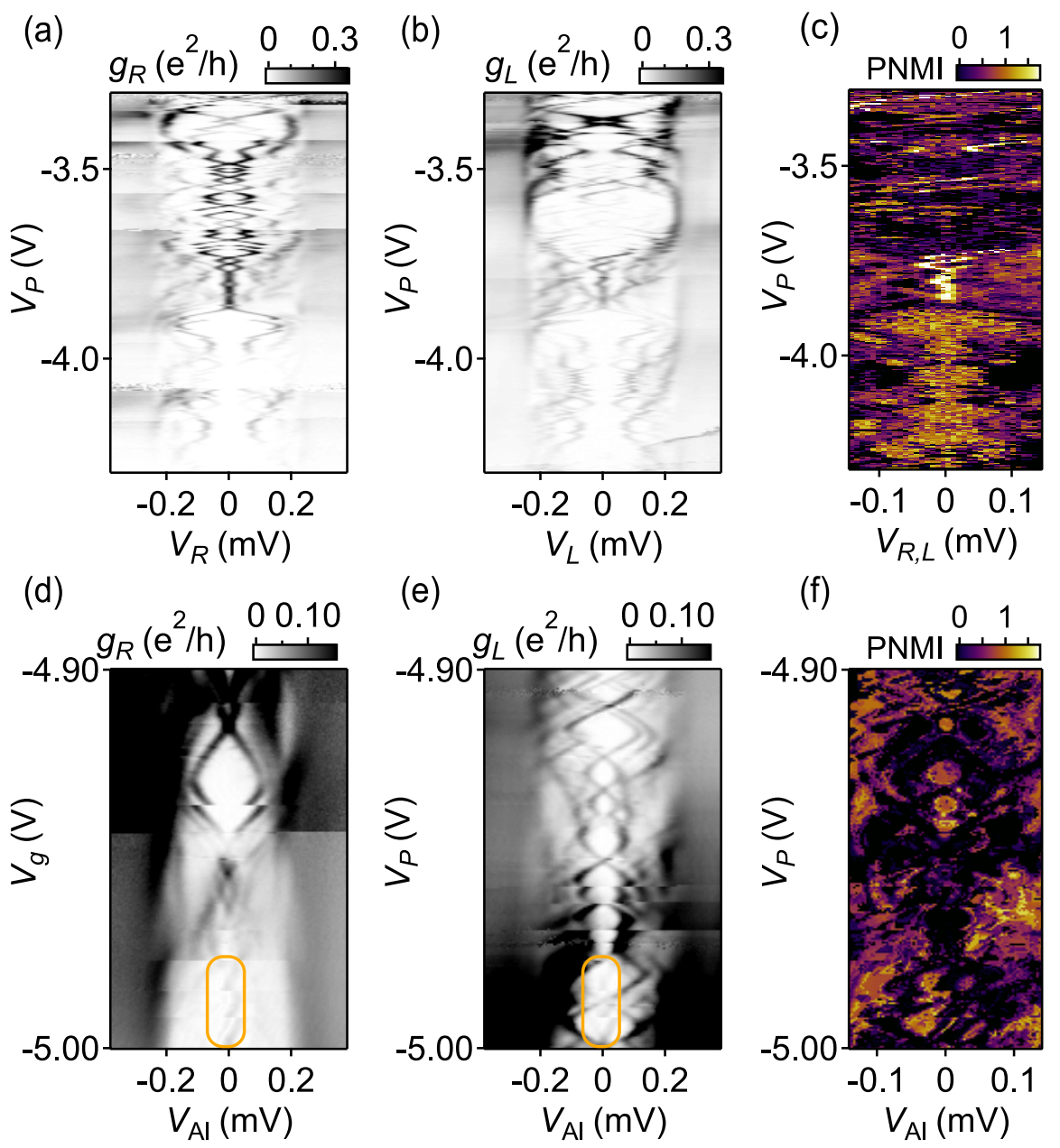

FIG. S3. (a) Short device right-conductance, $g_{R}$, measured as a function of gate, $V_{P}$ and bias, $V_{R}$. (b) Short device leftconductance, $g_{L}$, measured as a function of gate, $V_{P}$ and bias, $V_{L}$. (c) Short device conductance pointwise mutual information, $P M I$, mapped as a function of gate, $V_{P}$, and bias $V_{R, L}$ for subgap states. (d) Long device right-conductance, $g_{R}$, measured as a function of gate, $V_{P}$ and bias, $V_{\mathrm{Al}}$. Orange square indicates correlated region responsible for conductance peak in main text Fig. 3(d). (e) Long device left-conductance, $g_{L}$, measured as a function of gate, $V_{P}$ and bias, $V_{\mathrm{Al}}$. Orange squares indicates correlated region responsible for conductance peak in main text Fig. 3(d). (f) Long device conductance pointwise mutual information, $P M I$, mapped as a function of gate, $V_{P}$, and bias $V_{\mathrm{Al}}$ for subgap states.

in each bin $i$ is estimated as the number of samples in the bin divided by the total number of samples. The mutual information, MI, is then calculated as:

$$
\mathrm{MI}=\sum_{i=1}^{M} p\left(A_{i} \times B_{i}\right) \log \left(\frac{p\left(A_{i} \times B_{i}\right)}{p\left(A_{i} \times \mathbb{R}\right) p\left(\mathbb{R} \times B_{i}\right)}\right),
$$

where $p\left(A_{i} \times \mathbb{R}\right)$ is the probability density in the rectangle projected onto the left conductance axis, and $p\left(\mathbb{R} \times B_{i}\right)$ likewise projected onto the right conductance axis. The contribution of each bin in the sample space to the mutual information is known as the pointwise mutual information (PMI):

$$
\mathrm{PMI}=\log \left(\frac{p\left(A_{i} \times B_{i}\right)}{p\left(A_{i} \times \mathbb{R}\right) p\left(\mathbb{R} \times B_{i}\right)}\right) .
$$

The mutual information is the expected value of the pointwise mutual information over the entire distribution.

In the main text we quote the normalized mutual information, NMI, which lies in the range $[0,1]$ and is given by

$$
\mathrm{NMI}=\frac{\mathrm{MI}}{H},
$$


where $H$ is the Shannon entropy of the joint probability distribution:

$$
H=-\sum_{i=1}^{M} p\left(A_{i} \times B_{i}\right) \log p\left(A_{i} \times B_{i}\right) .
$$

The main text also quotes the normalized pointwise mutual information, NPMI, given by

$$
\mathrm{NPMI}=\frac{\mathrm{PMI}}{H} .
$$

Note that different normalization choices are possible, see e.g. [42].

The advantage of our selected method for calculating the mutual information is that the contribution of each bin can be easily assessed by visualizing the pointwise mutual information. This allows us to determine the relative contribution of the measured points to the total correlation, as shown by the maps of Fig. 4(e,f) in the main text. The drawback of the method, as with any method that relies on binning of the data, is the dependence of the calculated mutual information on the details of the binning. An alternative method for estimating the mutual information of the measured distribution is a non-parametric estimator, such as the one from [43]. This method relies only on the nearest-neighbor distances for estimating the mutual information. In Fig. S4 we compare the results obtained from the adaptive binning and the non-parametric algorithms, finding that they result in a similar numeric value. The calculations of the non-parametric estimator have been done using its implementation in the Python sklearn package[44].

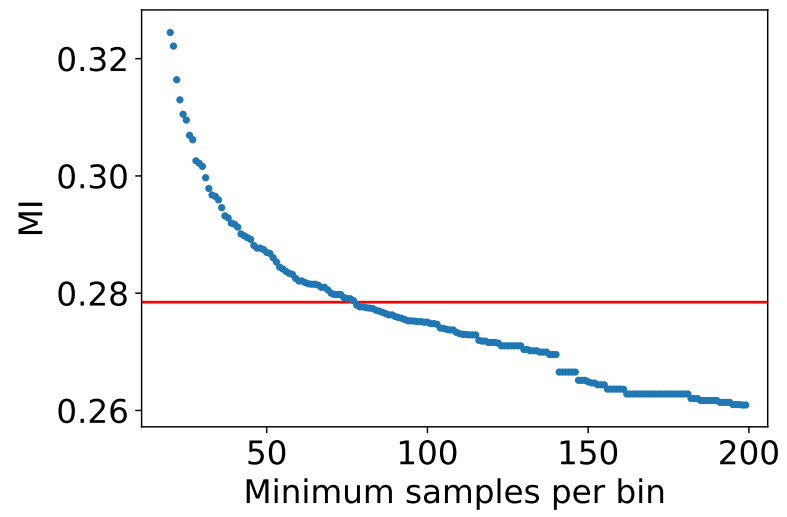

FIG. S4. Blue dots: mutual information (unnormalized) calculated with the adaptive binning method of [41] as a function of the minimum samples per bin cutoff for the subdivision algorithm. Red line: non-parametric estimate of the mutual information from [43].

\footnotetext{
* Equal contribution, lucas.casparis@microsoft.com
}

$\dagger$ Equal contribution, andrew.higginbotham@ist.ac.at 